\title{
BMJ Open Forty-five good things: a prospective pilot study of the Three Good Things well-being intervention in the USA for healthcare worker emotional exhaustion, depression, work-life balance and happiness
}

J Bryan Sexton, ${ }^{1,2}$ Kathryn C Adair ${ }^{1}$

To cite: Sexton JB, Adair KC. Forty-five good things: a prospective pilot study of the Three Good Things wellbeing intervention in the USA for healthcare worker emotional exhaustion, depression, work-life balance and happiness. BMJ Open 2019;9:e22695. doi:10.1136/ bmjopen-2018-022695

- Prepublication history and additional material for this paper are available online. To view these files, please visit the journal online (http://dx.doi. org/10.1136/bmjopen-2018022695).

Received 2 March 2018 Revised 24 January 2019 Accepted 5 February 2019

Check for updates

(C) Author(s) (or their employer(s)) 2019. Re-use permitted under CC BY-NC. No commercial re-use. See rights and permissions. Published by BMJ.

${ }^{1}$ Duke Center for Healthcare Safety and Quality, Duke University Health System, Durham, North Carolina, USA

${ }^{2}$ Psychiatry, Duke University School of Medicine, Durham, North Carolina, USA

Correspondence to Dr J Bryan Sexton; bryan.sexton@duke.edu

\section{ABSTRACT}

Objectives High rates of healthcare worker ( $\mathrm{HCW}$ ) burn-out have led many to label it an 'epidemic' urgently requiring interventions. This prospective pilot study examined the efficacy, feasibility and evaluation of the 'Three Good Things' (3GT) intervention for HCWs, and added burn-out and work-life balance to the set of wellbeing metrics.

Methods $228 \mathrm{HCWs}$ participated in a prospective, repeated measures study of a web-based 15-day long $3 \mathrm{GT}$ intervention. Assessments were collected at baseline and 1, 6 and 12-month post-intervention. The primary measure of efficacy was a derivative of the emotional exhaustion subscale of the Maslach Burnout Inventory. The secondary measures were validated instruments assessing depression symptoms, subjective happiness, and work-life balance. Paired samples t-tests and Cohen's d effect sizes for correlated samples were used to examine the efficacy of the intervention.

Results 3GT participants exhibited significant improvements from baseline in emotional exhaustion, depression symptoms and happiness at 1 month, 6 months and 12 months, and in work-life balance at 1 month and 6 months (effect sizes $0.16-0.52$ ). Exploratory subgroup analyses of participants meeting 'concerning' criteria at baseline revealed even larger effects at all assessment points $(0.55-1.57)$. Attrition rates were similar to prior 3GT interventions.

Conclusion 3GT appears a promising low-cost and brief intervention for improving HCW well-being.

Ethics and dissemination This study is approved by the Institutional Review Board of Duke University Health System (Pro00063703). All participants are required to give their informed consent prior to any study procedure.

\section{INTRODUCTION}

Healthcare worker (HCW) burn-out has reached a critical level. In the USA, over half of physicians and one-third of nurses report feeling burned out from their work. ${ }^{12}$ In recent years, HCWs have faced a staggering
Strengths and limitations of this study

- This pilot study examined the efficacy of the Three Good Things intervention for healthcare worker well-being over four time points: at baseline and three postintervention follow-ups (1 month, 6 months and 12 months).

- Efficacy was assessed with four well-being measures: emotional exhaustion, depression symptoms, subjective happiness and work-life balance.

- This pilot study is limited by not having a randomised control group. Results are limited to those who completed assessments.

- Attrition was high (only half of those who started went on to complete the 1-month postassessments) but similar to rates found in other comparable interventions.

number of increasing demands, including complex electronic medical records, new regulatory requirements, higher rates of student loan debt and increased production pressure. Simultaneously, HCWs are often not given the resources (or worse, are having their resources taken away) that are needed to meet these new demands. ${ }^{3}$ They report having less autonomy, less control over decision-making and less time to have meaningful interactions with patients. As demands increase and resources decrease, the result on any system or individual is strain, and that strain is what drives the increasing prevalence of burn-out. ${ }^{4}$ The increase is evident in the careful study by Shanafelt et al, detailing a $9 \%$ increase in physician burn-out between 2011 and $2014 .^{1}$

Maslach characterised burn-out as a syndrome of emotional exhaustion (EE), depersonalisation and reduced 
accomplishment that can occur among those who do 'people work' of some kind. ${ }^{5}$ Of particular interest to HCWs is EE, which refers to feelings of being overextended and exhausted by the emotional demands of one's work. Burn-out has severe negative consequences for both HCWs and patients. Burn-out has been linked to higher rates of HCW depression, substance abuse, anxiety and suicidal ideation, decreased immune system function, reduced marital satisfaction and greater risk of motor vehicle accidents. ${ }^{6-11}$ At an organisational level, burn-out has been connected to poorer relationships with patients and staff, higher rates of job turnover, earlier retirements and reduced productivity. ${ }^{12-14}$ Importantly, provider burn-out is also linked to serious patient safety outcomes, including higher rates of infection, increased medication errors, lower patient satisfaction, worse safety culture and higher standardised mortality ratios. ${ }^{15-19}$

To date, the literature has predominately focused on describing the prevalence of burn-out rather than testing interventions to reduce it. The most successful exception to this has been training in meditation called Mindfulness-Based Stress Reduction (MBSR), and similar meditation programmes that garner modest effect sizes. ${ }^{20-22}$ These programmes appear to be fairly effective, but they require a significant time commitment of approximately 8-10 weeks, 2.5 hours per week and 45-60 min of homework a day. Another promising intervention is the 'Colleagues Meeting to Promote and Sustain Satisfaction' (COMPASS) programme. ${ }^{23}$ It offers physicians 1 hour of protected time every 2 weeks to discuss the 'virtues and challenges of being a physician' over a meal paid for by the hospital (ie, this is not a low-cost intervention). A randomised clinical trial of COMPASS found that it leads to sustained improvements in burn-out and finding meaning in one's work. ${ }^{23} 24$

A recent meta-analysis of HCW interventions for burn-out revealed that, on average, interventions lead to small but significant improvements in burn-out. ${ }^{25}$ Interventions can be categorised as either aimed at helping individuals improve their burn-out, or organisational activities aimed to reduce perceived demands and/or increase resources. Organisational-level interventions, which tend to focus on improving workload, scheduling, communication, teamwork and changes in workflow, are generally more effective than individual-level interventions, which, to date, largely involve meditation training. ${ }^{25}$

Rather than ask busy and burned out HCWs to commit additional time and effort to learn a new skill like meditation during this intense time in their lives, or assume that hospital administration can be convinced to provide significant funding for well-being, we selected a significantly less time-consuming, but well validated psychological intervention called Three Good Things (3GT). ${ }^{26} 27$ 3GT is one of a family of positive interventions used as intentional activities aimed at cultivating positive cognitions and emotions. ${ }^{28}$ In other words, 3GT is thought to enhance participants' ability to notice genuinely positive things around them, and these can be the same things that were already present, but went unnoticed prior to the intervention.

3GT appears well suited for use with burned out populations due to research showing that burn-out reduces one's ability to notice and attend to positive stimuli. ${ }^{29}$ Moreover, 3GT has improved happiness and depressive symptoms in previous research. ${ }^{26}$ Burn-out and depression have been empirically and conceptually linked ${ }^{30}$ and share symptoms such as extreme exhaustion, feeling down and reduced performance. The 3GT intervention, however, has not been empirically evaluated with HCWs. 3GT involves a brief explanation, takes only a couple of minutes to complete and requires relatively little time or resources. In the resource-restricted environment of healthcare, such quick and low-cost strategies are welcome.

The primary aim of the current study was to test the efficacy of 3GT for HCW well-being through four metrics: burn-out (specifically, the EE component), which is the primary outcome of interest, as well as depression symptoms, subjective happiness and work-life balance. We hypothesised that 3GT participants would report reductions in EE and depressive symptoms, as well as improvements in subjective happiness and work-life balance, between baseline assessments and all three follow-up assessments.

Exploratory secondary aims of the study were to (1) test whether those who met 'per cent concerning' criteria at baseline exhibited stronger intervention effects, (2) examine whether baseline well-being was a predictor of completing or failing to complete follow-up assessments and (3) assess the feasibility and evaluation of the programme through attrition rates and participant evaluation.

\section{METHOD}

\section{Design and patient population}

This is a prospective pilot study of 3GT, conducted between February 2014 and March of 2015. HCWs enrolled over 4 weeks through a link on our centre's website (https:// www.hsq.dukehealth.org/), labelled '3GT February 2014.' Generally, people who seek the content on our website have a background in patient safety, quality improvement or well-being. There was also a brief explanation of the 3GT intervention during enrolment that provided an overview of the prevalence and severity of burn-out in healthcare, as well as the length and nature of the intervention as a method for 'noticing and recalling what is going well'. In this convenience sample, all HCWs (clinical and non-clinical) 18 and older were eligible to participate. This included physicians, registered nurses (RNs), nurse managers/charge nurses, physician assistant/nurse practitioners, hospital aides, physical therapists, occupational therapists, pharmacists, respiratory therapists, technologists/technicians, administrative support, other managers and students. Participants completed assessments at baseline, at 1 month (ie, 1 month after the last 
day of 3GT), 6 months and 12 months follow-up. Links to online assessments were sent to participants via email.

\section{Patient and public involvement}

Neither patients nor the public were involved in the current study.

\section{Measures}

To assess changes in well-being, we used four validated scales (EE, depression symptoms, subjective happiness and work-life balance) at each assessment point (see table 1 for Cronbach's alphas). These four metrics were selected a priori and they were the only metrics administered. Demographic questions were included at baseline and open-ended items were also included at the 1-month follow-up. In the 1-month assessment, we also asked for evaluation feedback on a 1-5 scale, ('strongly agree' to 'strongly disagree') through questions such as, 'I would recommend the 3 Good Things exercise to a friend,' 'I have encouraged others to try 3 Good Things,' 'I would like to participate in 3 Good Things again next year,' and with an open-ended comments text box.

In addition to using scale scores to examine change following 3GT, we also report the percentage of participants whose scores fell below well-being thresholds established by prior literature.

Burn-out EE Subscale: The Maslach Burnout Inventory, which is the gold-standard tool in the field of burn-out, has been used extensively with HCWs. A meta-analysis has revealed that of the three subscales (EE, depersonalisation and personal accomplishment), EE consistently produces the largest and most consistent coefficient alpha estimates, while depersonalisation and personal accomplishment were both lower and less consistent than EE. ${ }^{31}$ In addition to being more psychometrically robust, $\mathrm{EE}$ can be used to discriminate between burned out and non-burned out outpatients suffering from work-related neurasthenia (according to criteria outlined in the International Statistical Classification of Diseases and Related Health Problems - 10th Revision).$^{32}$ We used a 5-item derivative ${ }^{33}$ of the original 9-item EE scale. ${ }^{32}$ Example items include 'I feel frustrated by my job' and 'Events at work affect my life in an emotionally unhealthy way'. Participants respond using a 5-point Likert scale (1=disagree strongly; $5=$ agree strongly). Each participant's mean of the five items was converted to a $0-100$ point scale, with higher scores representative of more severe EE. A score of 50 or higher indicates that the respondent is not disagreeing with the EE statements, and is the first threshold for per cent concerning because it indicates at least mild EE. ${ }^{34}$ These 'per cent concerning' thresholds should not be considered clinically diagnostic, but rather identify those whose scores represent more of a struggle on a given metric. The per cent concerning threshold can be grasped quickly, providing an anchor for interpretation and offering a way of communicating something about the distribution of the data within a single number.
For EE, internal consistency in the current study was good (Cronbach's alpha $=0.85$ )

Depressive symptoms: The Center for Epidemiological Studies Depression Scale-10-item version is a psychometrically sound tool for screening respondents for clinical depression. ${ }^{35}$ All items are prefaced with, 'during the past week, how often did this occur' and includes items such as 'I could not 'get going' and 'my sleep was restless'. Responses are answered on a 4-point scale $(0=$ rarely or none; $3=$ all of the time). Each participant's responses are summed together to achieve a 0-30 point scale. A score of 10 or higher is considered a positive screen, and was the threshold we used to group participants by depressive symptom severity as 'per cent concerning. ${ }^{36}$ Internal consistency in the current study was good (Cronbach's alpha $=0.85$ )

Subjective Happiness: Lyubomirsky and Lepper's Subjective Happiness Scale (SHS) is a valid, psychometrically sound and internationally used scale of global happiness. ${ }^{37} 38$ Example items include 'In general I consider myself ( $1=$ nota very happy person to $7=$ avery happy person)' and 'Compared to most of my peers I consider myself (1=less happy to $7=$ =more happy)'. All four items of the SHS are answered using a 7-point scale, and each participant's responses were averaged, with higher scores representative of higher subjective happiness. Benchmarking data in diverse populations demonstrate mean SHS scores consistently flank a value of 5 on therefore a threshold of below 5 was used to group participants as the "per cent concerning. ${ }^{38}$ Internal consistency in the current study was very good (Cronbach's alpha=0.86).

Work-life balance: Work-life balance items are from the work-life climate scale, which has been shown to have good psychometrics when used on HCWs, ${ }^{39}$ Work-life balance items elicit behavioural work-life infractions by asking: During the past week, how often did this occur? Followed by phrases such as: Skipped a meal, arrived home late from work or slept less than 5 hours in a night. The response scale for the work-life climate items ranged from: rarely or none of the time (less than 1 day); some or a little of the time (1-2 days); occasionally or a moderate amount of time (3-4 days); all of the time (5-7 days) and not applicable. Work-life climate scale scores were computed by taking the mean of the items. Previous research has used 2 or fewer days as 'per cent positive,' referring to scores greater than 2 as work-life imbalance. We use the imbalance designation (per cent of respondents with a mean over 2 ) as the 'per cent concerning, ${ }^{39}$ Internal consistency in the current study was good (Cronbach's alpha $=0.81$ ).

\section{Good things intervention}

The original 3GT intervention encouraged participants to keep a log of things that go well for 7 days. ${ }^{26}$ We adapted 3GT to our HCW population to be simple and quick, but we modified it in several ways. First, we extended the length of the intervention to last 2 weeks (15 days), starting and ending on a Monday. In pilot 
Table 1 Respondent demographics of Three Good Things participants

\begin{tabular}{|c|c|c|c|c|}
\hline & $\begin{array}{l}\text { Day } 1 \\
\text { Participants }(n=228) \\
\text { n (\%) }\end{array}$ & $\begin{array}{l}\text { 1-month } \\
\text { postassessment } \\
(n=127) \\
n(\%)\end{array}$ & $\begin{array}{l}\text { 6-month } \\
\text { postassessment } \\
(\mathrm{n}=119) \\
\mathrm{n}(\%)\end{array}$ & $\begin{array}{l}\text { 12-month } \\
\text { postassessment } \\
(n=121) \\
n(\%)\end{array}$ \\
\hline \multicolumn{5}{|l|}{ Healthcare worker role } \\
\hline Admin support & $4(1.8)$ & $4(3.1)$ & $4(3.3)$ & $3(2.5)$ \\
\hline Attending/staff/fellow/resident physician & $31(13.6)$ & $22(17.3)$ & $19(16.0)$ & $22(18.2)$ \\
\hline Nurse manager/charge nurse & $15(6.6)$ & $13(10.2)$ & $13(10.7)$ & $9(7.4)$ \\
\hline Other* & $49(21.5)$ & $26(20.5)$ & $31(26.1)$ & 27 (22.3) \\
\hline Other manager (eg, clinic manager) & $52(22.8)$ & $26(20.5)$ & $21(17.4)$ & $24(19.8)$ \\
\hline Physician assistant/nurse practitioner & $12(5.3)$ & $4(3.1)$ & $4(3.3)$ & $6(5.0)$ \\
\hline Registered nurse & $55(24.1)$ & $25(19.7)$ & $21(17.4)$ & $24(19.8)$ \\
\hline Student & $7(3.1)$ & $4(3.1)$ & $5(4.1)$ & $5(4.1)$ \\
\hline Technologist/technician & $2(0.9)$ & $3(2.4)$ & $3(2.5)$ & $1(0.8)$ \\
\hline Missing & $1(0.4)$ & $0(0)$ & $0(0)$ & $0(0)$ \\
\hline \multicolumn{5}{|l|}{ Gender } \\
\hline Male & $39(17.1)$ & $25(19.7)$ & $22(18.5)$ & $24(19.8)$ \\
\hline Female & $186(81.6)$ & $102(80.3)$ & $96(80.7)$ & $96(79.3)$ \\
\hline Missing & $3(1.3)$ & $0(0)$ & $4(0.8)$ & $1(0.8)$ \\
\hline \multicolumn{5}{|l|}{ Shift } \\
\hline Days & $174(76.3)$ & $104(80.3)$ & $99(83.2)$ & $99(81.8)$ \\
\hline Evenings & $2(0.9)$ & $2(1.6)$ & $2(1.7)$ & $3(2.5)$ \\
\hline Nights & $16(7.0)$ & $8(6.3)$ & $3(2.5)$ & $4(3.3)$ \\
\hline Variable shifts & $35(15.4)$ & $13(10.2)$ & $15(12.6)$ & $15(12.4)$ \\
\hline Missing & $1(0.4)$ & $0(0)$ & $0(0)$ & $0(0)$ \\
\hline \multicolumn{5}{|l|}{ Years of experience total } \\
\hline$<1$ year & $7(3.1)$ & $2(1.6)$ & $3(2.5)$ & $1(0.8)$ \\
\hline $1-4$ years & $17(7.4)$ & $7(5.5)$ & $10(8.4)$ & $8(6.6)$ \\
\hline 5-10years & $34(14.9)$ & $22(17.3)$ & $15(12.6)$ & $17(14.0)$ \\
\hline 11-20years & $56(24.6)$ & $32(25.2)$ & $30(25.2)$ & $28(23.1)$ \\
\hline $21+$ years & $110(48.2)$ & $64(50.0)$ & $61(50.4)$ & $67(55.4)$ \\
\hline Missing & $4(1.8)$ & $0(0)$ & $0(0)$ & $0(0)$ \\
\hline \multicolumn{5}{|l|}{ Years in current position } \\
\hline$<1$ year & $30(13.1)$ & $17(13.4)$ & $16(13.4)$ & $16(13.2)$ \\
\hline $1-4$ years & $70(30.7)$ & $43(33.9)$ & $44(37.0)$ & $38(31.4)$ \\
\hline 5-10years & $56(24.6)$ & $28(22.0)$ & $23(19.3)$ & $24(19.8)$ \\
\hline 11-20years & $45(19.7)$ & $20(15.7)$ & $18(15.1)$ & $24(19.8)$ \\
\hline $21+$ years & $26(11.4)$ & $19(15.0)$ & $18(15.1)$ & $19(15.7)$ \\
\hline Missing & $1(0.4)$ & $0(0)$ & $0(0)$ & $0(0)$ \\
\hline \multicolumn{5}{|l|}{ Scale reliabilities (Cronbach's alpha) } \\
\hline Emotional exhaustion† (5 items) & 0.85 & 0.85 & 0.87 & 0.87 \\
\hline Depression symptoms $\ddagger$ (10 items) & 0.85 & 0.79 & 0.84 & 0.81 \\
\hline Subjective happiness§ (4 items) & 0.86 & 0.86 & 0.87 & 0.88 \\
\hline Work-life Balance (7 items) & 0.81 & 0.81 & 0.79 & 0.77 \\
\hline
\end{tabular}

*This category includes the positions clinical social worker, clinical support (certified medical assistant, nurses aid, etc), dietician/nutritionist, occupational therapist, respiratory therapist and those who selected 'other'.

†Emotional exhaustion was assessed with a 5-item derivative of the emotional exhaustion subscale of the Maslach Burnout Inventory.

‡Depression symptoms were measured with the Center for Epidemiological Studies Depression Scale.

§Subjective Happiness Scale was assessed with Lyubomirsky and Lepper's Subjective Happiness Scale.

IWork-life balance was assessed with the work-life climate scale. Higher scores reflect higher levels of each construct, with the exception of work-life balance, in which case lower numbers reflect healthier work-life balance.

testing, we found that more people complete the full 2 weeks if they start on a Monday. Second, using Qualtrics survey software, we sent nightly email reminders to enter
'What went well today, and what was your role in making it happen?' in three text boxes. Third, to induce a brief savouring period, they were instructed to select a positive 
emotion that best fit how they felt about their good thing, from a drop-down list of 10 positive emotions (eg, Joy, Gratitude, Hope, etc) with options for 'not applicable' and 'other'. ${ }^{40}$ Prior to submitting their responses, participants could choose to anonymously share their good things with other participants in the following night's log. All participants, on completion of their log, were automatically redirected to the log of good things participants submitted and approved for sharing the day before. This exposure to shared good things was another opportunity for savouring.

\section{Statistical analysis}

Cronbach's alpha was used to assess the internal reliability of the scales (values above 0.70 are considered acceptable, 0.80 good and 0.90 very good). ${ }^{41}$ Feasibility of 3GT for HCWs was assessed through attrition (uptake) and participant evaluations. Independent samples t-tests examined whether baseline levels of the well-being metrics predicted dropping out of the study at the 1,6 and 12-month follow-ups. Paired samples t-tests were used to test for improvement in the well-being variables across the baseline and 1, 6 and 12-month follow-ups. These analyses used listwise deletion for missing data. Participants who were lost to follow-up were still included in any analysis for which they had provided data. For all analyses, $p$ values less than 0.05 were considered significant and all tests were two tailed. A power analysis indicated that our sample size provided high power $(1-\beta)>0.9$ to detect relatively small effect sizes (Cohen's $\mathrm{d}=0.3$ ). Cohen's d effect sizes for correlated samples were computed using the formula $\mathrm{d}=\mathrm{M}_{\text {diff }} / \mathrm{SD}_{\text {pooled }}$. Generally, effect sizes around 0.2 are considered small, 0.5 are considered medium, 0.8 large, 1.2 very large and 2.0 huge ${ }^{42}$ Analyses were performed using IBM SPSS software V.24.

\section{RESULTS}

\section{Respondent demographics}

Two hundred and twenty-eight HCWs (81\% female, $76.3 \%$ day shift) participated in at least day 1 of the 3GT intervention, and $55(24.6 \%)$ of them identified as RNs. See table 1 for demographic information. Of these, 127 participants completed the 1-month follow-up survey (55.7\% of those who completed 3GT day $1 ; 80.3 \%$ were female and $19.7 \%$ identified as nurses). One hundred and nineteen participants completed the 6-month follow-up survey (52.2\% of those who completed 3GT day $1 ; 80.7 \%$ were female and $17.4 \%$ identified as nurses). One hundred and twenty-one participants completed the 12-month follow-up (53.1\% of those who completed 3GT day $1 ; 79.3 \%$ were female and $19.8 \%$ identified as nurses). Participants in the 'other' role category most commonly identified as working in roles with clinical exposure not captured elsewhere (eg, occupational therapist, physical therapist, pharmacist, long-term care), patient safety or quality, administration, counselling and psychological services, and patient revenue management.

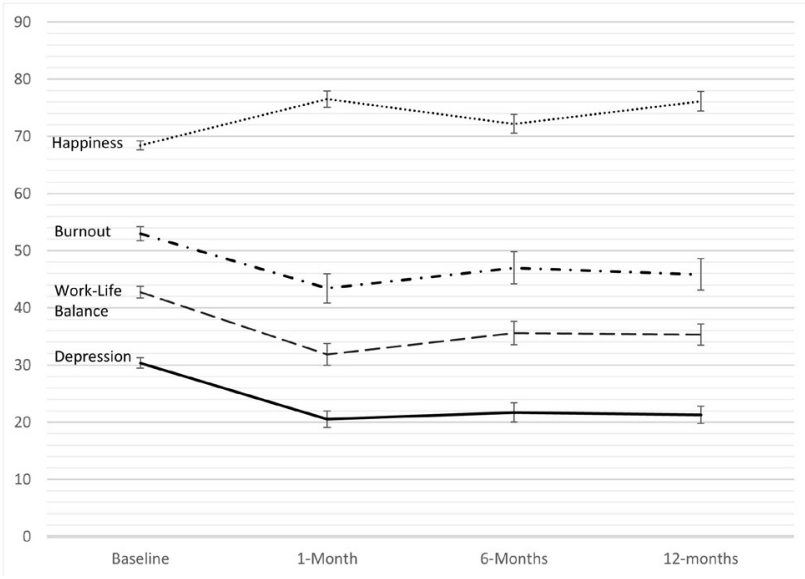

Figure 1 Well-being metrics at baseline, 1, 6 and 12-month follow-up. For demonstration purposes, all variables were scaled 0-100 in this graph. Burn-out was already scaled 0-100, depression scores (response range 0-30) were multiplied by 3.33 , WLB scores (response range 1-4) were subtracted by 1 and multiplied by 33.33 and Subjective Happiness Scores (response range 1-7) were subtracted by 1 and multiplied by 16.66. WLB, work-life balance.

Of those who participated, $48.2 \%$ reported 21 or more years of experience in their profession. The most common response for the number of years in current position was $1-4$ years.

\section{Well-being metrics over time}

Internal reliabilities are reported at the bottom of table 1. Internal consistency for each metric was good at each time point, with Cronbach alphas ranging from 0.77 to 0.87 . All participant well-being metrics were relatively highly correlated with themselves across time, especially Subjective Happiness, with correlations between 0.74 and 0.80 . Figure 1 illustrates mean rates of all scales at each time point.

Independent t-tests assessed whether baseline wellbeing metrics predicted dropping out or not at each follow-up assessment. At the 1-month follow-up, baseline levels of EE did not predict dropping out (see table 2), however, participants higher in problems with work-life balance, depression and lower in happiness were less likely to complete 1-month assessments. None of the baseline measures predicted dropping out at the 6 or 12-month mark.

Table 3 shows the means and results of the analyses across the whole sample, as well as the subgroup of respondents reporting concerning levels of each well-being metric at each assessment point. Paired t-tests conducted on the entire sample revealed significant improvement, $\mathrm{ps}<0.05$, in EE, depression symptoms and happiness at 1,6 and 12 months, and in work-life balance at 1 and 6 months. Effect sizes across all four metrics were small to medium ranging from 0.16 to 0.52 . Exploratory subgroup analyses on participants who met 'per cent concerning' criteria at baseline also revealed significant improvement across assessments, $\mathrm{ps}<0.05$, as well as moderate to 
Table 2 Independent t-tests comparing baseline well-being for those who dropped out of 1, 6 and 12-month follow-up assessments

\begin{tabular}{|c|c|c|c|c|c|c|}
\hline \multirow[b]{4}{*}{ Baseline Well-being Metric } & \multicolumn{2}{|c|}{1 month } & \multicolumn{2}{|c|}{6 months } & \multicolumn{2}{|c|}{12 months } \\
\hline & \multirow{2}{*}{$\begin{array}{l}M \\
S D \\
\end{array}$} & \multirow[b]{3}{*}{$95 \% \mathrm{Cl}$} & \multirow{2}{*}{$\begin{array}{l}M \\
S D \\
\end{array}$} & \multirow[b]{3}{*}{$95 \% \mathrm{Cl}$} & \multirow{2}{*}{$\begin{array}{l}\mathrm{M} \\
\mathrm{SD}\end{array}$} & $\mathbf{T}$ \\
\hline & & & & & & \\
\hline & $\mathbf{n}$ & & $\mathbf{n}$ & & $\mathbf{n}$ & $95 \% \mathrm{Cl}$ \\
\hline \multicolumn{7}{|l|}{ Emotional exhaustion $†$} \\
\hline \multirow[t]{3}{*}{ Retained } & 49.67 & 1.56 & 56.46 & -1.57 & 52.42 & 0.25 \\
\hline & 27.46 & (-9.86 to 1.14$)$ & 27.68 & $(-1.17$ to 10.15$)$ & 27.73 & $(-6.35$ to 4.90$)$ \\
\hline & 127 & & 119 & & 121 & \\
\hline \multirow[t]{3}{*}{ Dropped out } & 54.03 & & 51.97 & & 53.15 & \\
\hline & 27.53 & & 27.49 & & 27.53 & \\
\hline & 400 & & 408 & & 406 & \\
\hline \multicolumn{7}{|l|}{ Depression symptoms $\ddagger$} \\
\hline \multirow[t]{3}{*}{ Retained } & 8.12 & $2.08^{\star}$ & 9.42 & -0.6 & 8.54 & 1.15 \\
\hline & 5.35 & $(-2.47$ to -0.15$)$ & 5.93 & $(-0.88$ to 1.68$)$ & 5.51 & $(-1.95$ to 0.47$)$ \\
\hline & 115 & & 106 & & 108 & \\
\hline \multirow[t]{3}{*}{ Dropped out } & 9.43 & & 9.02 & & 9.28 & \\
\hline & 6.04 & & 5.9 & & 6 & \\
\hline & 359 & & 368 & & 366 & \\
\hline \multicolumn{7}{|l|}{ Subjective Happiness§ } \\
\hline \multirow[t]{3}{*}{ Retained } & 5.28 & $-1.97^{\star}$ & 5.04 & 0.81 & 5.25 & -1.59 \\
\hline & 1 & (0.01 to 0.43 ) & 1.17 & $(-0.32$ to 0.14$)$ & 1.07 & $(-0.04$ to 0.40$)$ \\
\hline & 127 & & 119 & & 121 & \\
\hline \multirow[t]{3}{*}{ Dropped out } & 5.06 & & 5.13 & & 5.07 & \\
\hline & 1.11 & & 1.06 & & 1.09 & \\
\hline & 402 & & 410 & & 408 & \\
\hline \multicolumn{7}{|l|}{ Work-life Balanceף } \\
\hline \multirow[t]{3}{*}{ Retained } & 2.17 & $2.09^{*}$ & 2.24 & 0.83 & 2.21 & 1.36 \\
\hline & 0.7 & $(-0.21$ to -0.08$)$ & 0.63 & $(-0.19$ to 0.07$)$ & 0.69 & $(-0.23$ to 0.05$)$ \\
\hline & 126 & & 118 & & 120 & \\
\hline \multirow[t]{3}{*}{ Dropped out } & 2.32 & & 2.3 & & 2.3 & \\
\hline & 0.7 & & 0.71 & & 0.7 & \\
\hline & 400 & & 408 & & 406 & \\
\hline
\end{tabular}

${ }^{*} \mathrm{P}<0.05$.

†Emotional exhaustion was assessed with a 5-item derivative of the emotion exhaustion subscale of the Maslach Burnout Inventory. $\ddagger$ Depression symptoms were measured with the Center for Epidemiological Studies Depression Scale.

$\S$ Subjective Happiness Scale was assessed with Lyubomirsky and Lepper's Subjective Happiness Scale.

ףWork-life balance was assessed with the work-life climate scale. Higher scores reflect higher levels of each construct, with the exception of work-life balance, in which case lower numbers reflect healthier work-life balance.

very large effect sizes $(0.55-1.57)$. Finally, we examined whether the number of completed 3GTs days predicted changes in well-being across the study. We computed wellbeing change scores for all metrics between baseline and follow-up assessments, and used Spearman correlations to detect associations between change scores and number of completed 3GTs days. No significant associations were found, $\mathrm{ps}>0.05$.

\section{HCW 3GT feasibility and participant experience}

Two hundred and twenty-eight participants engaged in day 1 of 3GT. Of these, the average number of 3GTs days was $8.96(\mathrm{SD}=4.74)$. Although attrition may have occurred for a variety of reasons, the only reason participants volunteered was not having time to complete surveys. $74.4 \%$ of participants selected the option to share their 3GT responses with other participants at least once, 


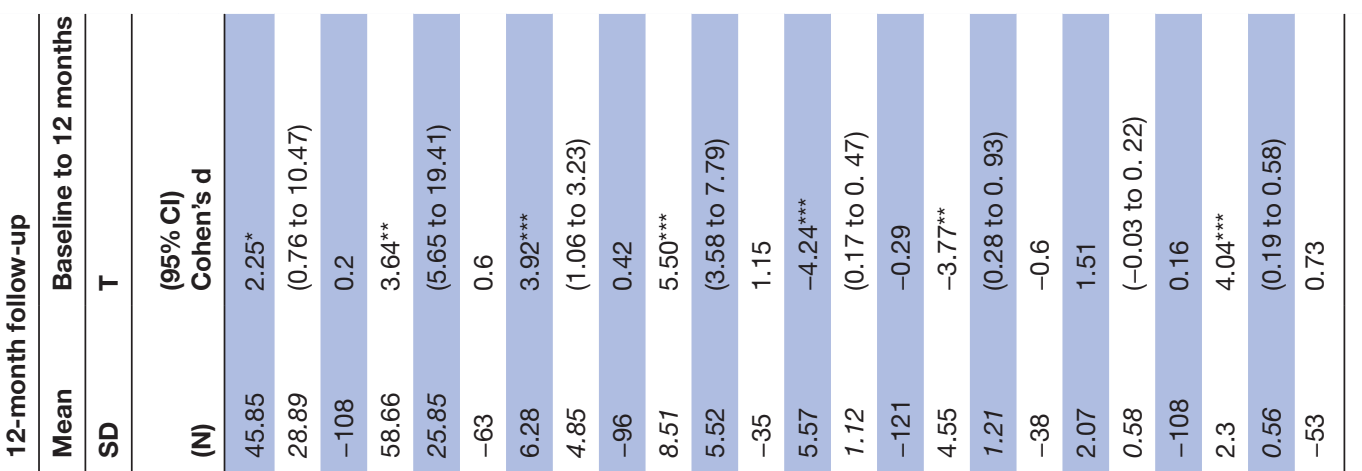

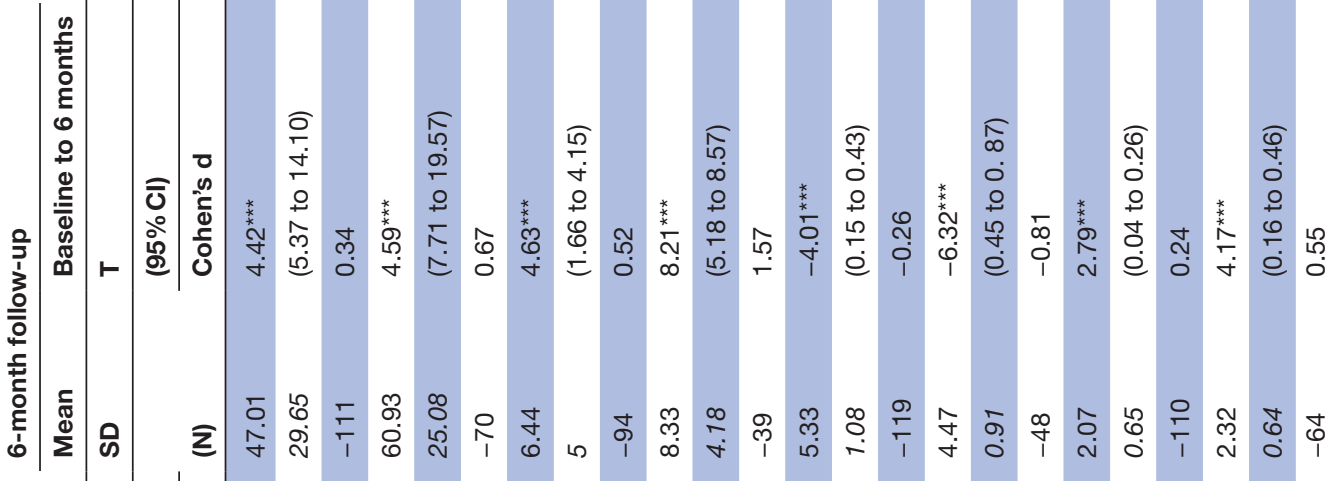

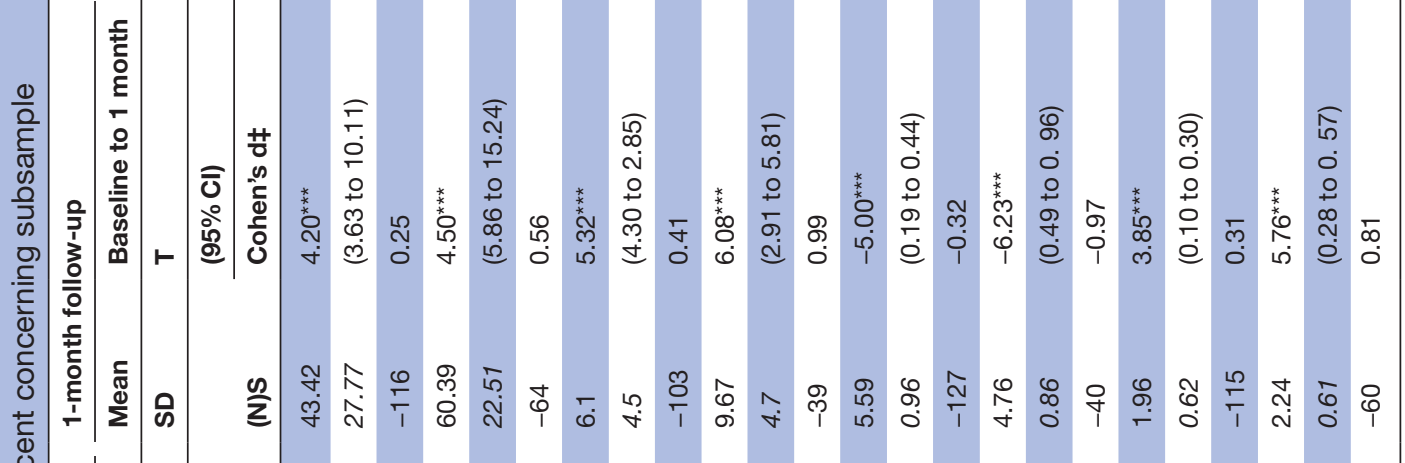
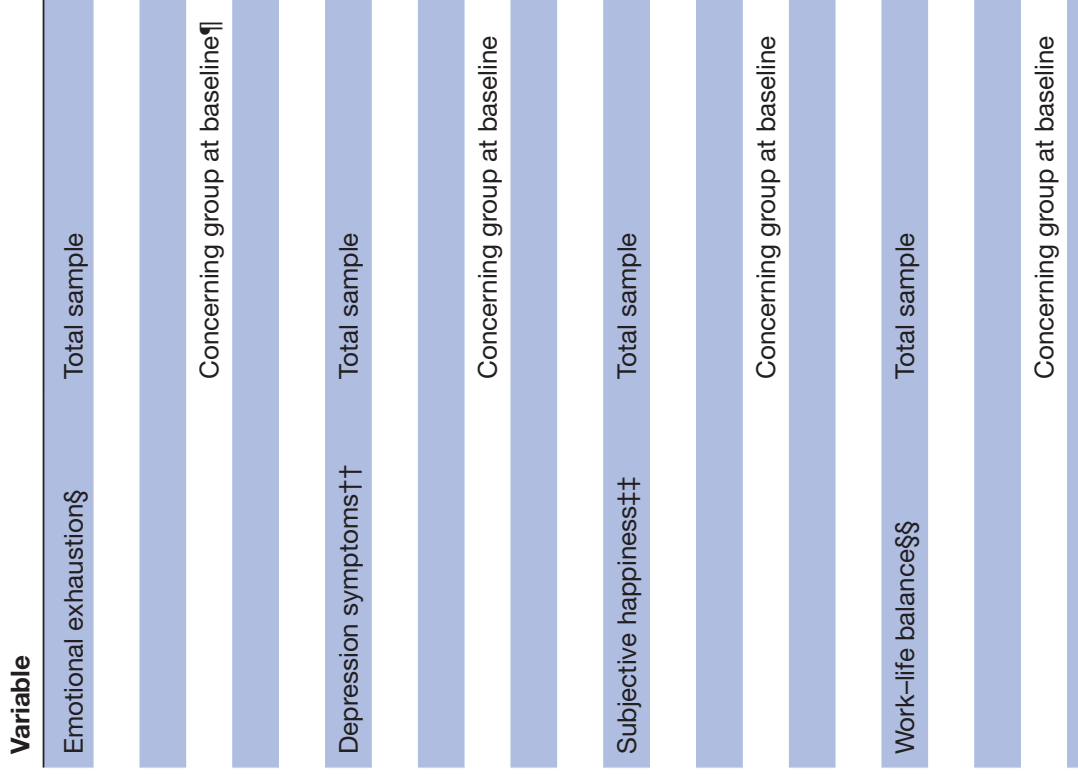
and rates of sharing remained fairly stable across the days (mean $=70.9 \%$, range $74.4 \%-69.1 \%)$. 3GT submissions included a wide variety of good things that happened both in and outside of work. A sample of de-identified 3GT submissions can be found in the online supplementary appendix. Participant evaluations of 3GT were overwhelmingly positive: $95.8 \%$ agreed with 'I would recommend the 3 Good Things exercise to a friend.' $85.3 \%$ agreed with 'I have encouraged others to try 3 Good Things.' And $92.7 \%$ agreed with 'I would like to participate in 3 Good Things again next year.'

Some participants also offered feedback in an openended text box. Representative comments can be found in the online supplementary appendix, and included: 'Some nights I really felt as if I didn't have a good day. Three good things helped me focus on the fact that even if the day wasn't my best there were still good things about it.' 'I love this format. It is so obstacle-free, yet just enough to really REGISTER and acknowledge tangible good things each day. Reading others' good things makes me realize how many of those things (or similar) also happened to me, and feels like it amplifies my good feelings and sense of contentment, gratitude, happiness.' 'Pushes you to look for the good all day long!'

\section{DISCUSSION}

The current pilot study adapted and tested the 3GT intervention for use by HCWs. Participants of our nightly web-based intervention exhibited improvement across all four of our well-being metrics: burn-out (EE), depression, problems with work-life balance and happiness. Three of these metrics showed significant improvement between baseline and the 12-month follow-up, and the fourth metric, problems with work-life balance, showed improvement between baseline and the 1 and 6-month follow-ups. 3GT is a relatively simple, brief and low-cost intervention. To our knowledge, this is the first study demonstrating the efficacy of 3GT in HCWs across variety of well-being measures, including EE.

Similar to previous work, half of our sample reported at least mild EE at baseline, and 37.2\% reported symptoms of depression. ${ }^{1}$ Rates of EE and depression in our sample were significantly lower a full year after participating in 3GT. Of note is the percentage of those who met the threshold for a positive screen for depression was cut approximately in half at 1 month (from $37.2 \%$ to $19.1 \%$ ). In fact, participants who met the concerning thresholds at baseline for each of the well-being metrics exhibited particularly strong improvements. Cohen's d effect sizes for all measures across the whole sample ranged from 0.16 to 0.52 and from 0.55 to 1.57 in the per cent concerning at baseline group.

Given how brief and simple it is to participate in a 3GT intervention, these effect sizes are notable in comparison to current psychological interventions. A meta-analysis of selective serotonin reuptake inhibitors (SSRIs) revealed a relatively small effect size $(\mathrm{d}=0.32$ between SSRIs and a placebo),${ }^{43}$ whereas the effect sizes in the current study approach those found by Krasner et al, in their widely cited study of MBSR for HCWs (0.62 for EE, 0.55 for depression). This is notable considering the differential time commitment involved in participating in 3GT compared with meditation training or an MBSR course. There may be a high return on investment in brief, simple and accessible interventions like 3GT.

The study also tested whether baseline levels of wellbeing predicted completion of the follow-up assessments. Baseline levels of EE did not predict dropping out of any of the follow-up assessments; however, those who dropped out at the 1-month assessment mark had higher levels of depression, problems with work-life balance and lower levels of happiness at baseline. Baseline well-being did not predict completing or dropping out of the 6 or 12-month assessments. These results indicate that at least some wellness indices appear to have short-term predictive power for who is at risk of dropping out. However, in concert with the 'per cent concerning' findings, these results suggest that if someone who is really struggling at baseline is able to power through and complete the intervention, he/she is likely to experience an even greater benefit than those with relatively higher well-being at baseline. These findings complement prior work indicating that those with worse well-being (ie, lower positive affect, clinically depressed) appear to benefit the most from positive psychology interventions. ${ }^{28} 442844$

Relieving HCW burn-out is a national imperative. Not only is burn-out associated with devastating provider outcomes like substance abuse and suicidal ideation, ${ }^{78}$ it is also linked to consequential patient safety outcomes, including medication errors and standardised mortality ratios. ${ }^{16}$ Existing interventions for burn-out largely centre on learning mindfulness-based meditation. Meditation training appears helpful for HCW burn-out, ${ }^{20}$ however, in addition to the time commitment required, it is not broadly appealing to many providers due to religious, logistical or personal reasons. In other words, MBSR appears beneficial, it just is not the right fit for many.

Since 2001, the job demands-resources model has accurately and repeatedly demonstrated that increasing demands while decreasing resources creates strain on the workforce. ${ }^{45}$ This strain has been called burn-out, low engagement, compassion fatigue, low safety culture and other similar monikers. Rigorous psychological research has consistently shown that experiencing positive emotions is associated with the ability to recover after emotional upheavals. ${ }^{46}$ Positive emotions, like hope, gratitude and serenity, help people build personal resources, such as social bonds, intellectual skills and motivation for personal growth. ${ }^{47}$ Positive emotions also have the ability to recharge our self-regulation when we are feeling depleted $^{48}$ and to undo the cardiovascular after-effects of an emotional upheaval, relative to negative emotions and control groups. ${ }^{47}$ When access to positive emotion 'engines' is interrupted, through new or increasing 
demands, we end up going to work each day with weaker and weaker batteries. This is not weakness on the part of the person, it is the failure of our healthcare systems to create environments that promote well-being in the workforce.

By merging the well-supported theories of positive emotion ${ }^{49}$ and of job demands-resources, ${ }^{45}$ we propose that an actionable approach to burn-out should consider the restorative effects of positive emotions. The current intervention, which involved savouring positive emotions, found improvement across four well-being metrics, suggesting that there is something fundamental about positive emotions that the field might be missing in the way that we traditionally view burn-out. Incorporating the role of positive emotions (or the lack of them) into the field's understanding of burn-out may expedite the development of effective burn-out interventions that include the mechanism of positive emotions, as well as improve our understanding the sources of burn-out. Rather than focusing on the increasing experience of negative emotions in burn-out, perhaps we should also focus on the reduced experience of restorative positive emotions.

Prior research has found that those experiencing burn-out are more likely to attend to negative stimuli, even at the expense of not attending to positive stimuli that could improve one's mood..$^{29}$ By having HCWs report nightly on 3GT that happened that day, they might be recalibrating their emotional well-being through more frequent experiences of positive emotions, as well as expanding their awareness of positive events. Although the mechanisms of action of this tool have yet to be definitively examined, anecdotal evidence suggests two possibilities. The first is that at the moment of reporting, there is savouring of positive emotion, whereby participants re-experience the positive emotions that accompanied the good thing that happened to them, perhaps also with a broader appreciation for that experience and what it involved. This can occur while entering the good thing, or selecting the positive emotion that goes with it, or when reading the good things in the shared logs. The second possible mechanism is that by knowing that they will be reporting on 3GT each evening, participants become more aware of the good things happening around them. A few participants commented that this was their experience, even at times thinking, 'This can be one of my three good things I'll write about tonight!'

3GT as a resiliency tool has several strengths. The first is that the tool itself has little to no costs to deploy. We used a web-based format, but it is effective when administered as a paper-based intervention. ${ }^{26}{ }^{27}$ Further, completion of the tool requires minimal time commitment from the participants; it takes approximately 3 min to complete each day. The tool is straightforward as well, requiring no additional skills training, and we found that it was applicable to everyone regardless of workplace role. Participants submit a wide variety of 'good' things each night, including events that occurred inside and outside of the workplace. A wide variety of 3GT submissions have been identified by qualitative researchers as generally fitting under one of three overarching themes: having a good day at work, having supportive relationships and making meaningful use of self-directed time. ${ }^{50}$ The low cost, minimal time and effort commitment, and widely enjoyable nature of 3GT are attractive features for burned out providers and organisations looking for ways to address the urgent need to improve well-being.

The current pilot study has several strengths. The study examined the efficacy of a web-based 3GT intervention for HCWs across a wide variety of roles in healthcare. Wellbeing metrics were assessed across four time points: at baseline and three postintervention follow-ups (1 month, 6 months, 12 months), using four validated measures of well-being. We reported results on a 100-point scale, as well as used per cent concerning, and included effect sizes.

Our study has several limitations. First, the study was a non-randomised pilot, and there was not a control group, which precludes any discussion of causality. Rather, we can only say that participants who signed up for 3GT and completed follow-up assessments reported significant improvements across all outcomes. It is possible that participants who engaged in 3GT but did not complete postassessments did not benefit to the same degree as those who did complete post assessments. Without a control group, we also cannot say that the results we found were not due, in part or in whole, to expectancy or placebo effects. Nevertheless, previous 3GT studies have used control groups to demonstrate that the 3GT intervention is responsible for the changes in well-being. ${ }^{26}{ }^{27} \mathrm{We}$ note also that our sample is composed largely of women (81\% of participants at baseline), however, this is generally in line with base rates of women working in healthcare in the USA (in 2017, $75 \%$ of HCWs were female). ${ }^{51}$ Future research should oversample for men and examine whether 3GT is equally effective across genders.

Second, our study also suffered from attrition in that only $56 \%, 52 \%$ and $53 \%$ of those who completed day 1 3GT completed the 1, 6 and 12-month assessments, respectively. This rate of attrition is similar to other comparable studies. ${ }^{27} 5253$ In a methodologically rigorous study by Gander et al, only $67.3 \%$ of those assigned to an intervention carried out the intervention, and only $38.9 \%$ completed all follow-up assessments. ${ }^{27}$ In light of these attrition rates, it should be noted that people are notoriously bad at maintaining self-improvement goals. Even among people seeking treatment for obesity using weight loss programmes in medical centres, one-third had to half discontinue their programme and are lost to follow-up. ${ }^{54} 5$ Similarly, over $40 \%$ of people seeking treatment through a smoking cessation clinic were lost to follow-up, ${ }^{56}$ and another $48 \%$ of web-based smoking cessation individuals are lost to follow-up. ${ }^{57}$ Attrition is a primary barrier to evaluating web-based interventions, with levels often reaching $60 \%-80 \% .^{58}$

It is unclear from our study whether the effects observed were the result of the intervention alone. Intrinsic 
characteristics of the cohort that completed follow-up assessments may have predisposed them to improvement in EE and depression. Still, prior randomised controlled studies of 3GT for 1 week (a smaller dose) have demonstrated improvement with this intervention, which we believe extends to our results. ${ }^{53}$

We note also that those who needed this intervention the most (ie, those with higher burn-out and depression, and worse work-life balance at baseline) were more likely to drop out of the study before the 1-month follow-up (after which time attrition did not change much). For those initially overwhelmed individuals, taking the time to engage in 3GT still felt like 'one more thing'. Future research should note this specific need to maintain participant focus during the early stages of studies of this kind, by making the tool more accessible, enjoyable, meaningful and brief.

Third, 3GT is an intervention designed to help individuals build their well-being resources, but does not address the demands placed on them personally or professionally. The pace and intensity of contemporary healthcare are what causes the strain that leads to impaired well-being. 3GT, while promising as one simple tool that should be part of a much larger toolkit, does not prevent future sources of strain. In the long term, a family of valid and accessible interventions need to be targeted at both facilitating individual well-being ('levers that I can pull for myself right now') and preventing problems of strain ('levers that my institution/work setting pull for me to provide additional resources that prevent and alleviate strain').

Despite these limitations, this pilot study expands on previous research by demonstrating that a modification of 3GT for HCW appears to offer a simple and effective intervention to improve emotional well-being and decrease EE. Current research is underway to evaluate the efficacy of 3GT with respect to the endurance of the improvements, to examine mechanisms of action and to use control groups.

\section{CONCLUSIONS}

Given the relationship between HCW well-being and healthcare quality, current findings that well-being metrics appear to improve across repeated measures are encouraging. 3GT has now been demonstrated as a low-cost strategy to potentially improve well-being, such that refinement and extension of this type of intervention is warranted.

Contributors JBS developed the procedure, collected the data, contributed to analyses and cowrote the paper. KCA contributed to analyses and cowrote the paper.

Funding This work was supported by NIH grant number R01 HD084679-01.

Competing interests None declared.

Patient consent for publication Not required.

Ethics approval This study was approved by our institutional review board (Study Protocol ID: Pro00063703).
Provenance and peer review Not commissioned; externally peer reviewed.

Data sharing statement The authors will share survey data from the study on reasonable request to the first author.

Open access This is an open access article distributed in accordance with the Creative Commons Attribution Non Commercial (CC BY-NC 4.0) license, which permits others to distribute, remix, adapt, build upon this work non-commercially, and license their derivative works on different terms, provided the original work is properly cited, appropriate credit is given, any changes made indicated, and the use is non-commercial. See: http://creativecommons.org/licenses/by-nc/4.0/.

\section{REFERENCES}

1. Shanafelt TD, Hasan O, Dyrbye LN, et al. Changes in burnout and satisfaction with work-life balance in physicians and the general US working population between 2011 and 2014. Mayo Clin Proc 2015;90:1600-13.

2. Aiken LH, Sermeus W, Van den Heede K, et al. Patient safety, satisfaction, and quality of hospital care: cross sectional surveys of nurses and patients in 12 countries in Europe and the United States. BMJ 2012;344:e1717.

3. Bakker AB, Demerouti E. The Job Demands-Resources model: state of the art. Journal of Managerial Psychology 2007;22:309-28.

4. Bakker AB, Demerouti E. Job demands-resources theory: taking stock and looking forward. J Occup Health Psychol 2017;22:273-85.

5. Maslach C. Burnout: The Cost of Caring. New York: Prentice-Hall, 1982.

6. Wurm W, Vogel K, Holl A, et al. Depression-Burnout Overlap in Physicians. PLoS One 2016;11:e0149913.

7. Pedersen AF, Sørensen JK, Bruun NH, et al. Risky alcohol use in Danish physicians: associated with alexithymia and burnout? Drug Alcohol Depend 2016;160:119-26.

8. van der Heijden F, Dillingh G, Bakker A, et al. Suicidal thoughts among medical residents with burnout. Arch Suicide Res 2008;12:344-6.

9. Mommersteeg PM, Heijnen CJ, Kavelaars A, et al. Immune and endocrine function in burnout syndrome. Psychosom Med 2006;68:879-86.

10. Golub JS, Weiss PS, Ramesh AK, et al. Burnout in residents of otolaryngology-head and neck surgery: a national inquiry into the health of residency training. Acad Med 2007;82:596-601.

11. West CP, Tan AD, Shanafelt TD. Association of resident fatigue and distress with occupational blood and body fluid exposures and motor vehicle incidents. Mayo Clin Proc 2012;87:1138-44.

12. Ramirez AJ, Graham J, Richards MA, et al. Mental health of hospital consultants: the effects of stress and satisfaction at work. Lancet 1996;347:724-8.

13. Dewa CS, Jacobs $P$, Thanh NX, et al. An estimate of the cost of burnout on early retirement and reduction in clinical hours of practicing physicians in Canada. BMC Health Serv Res 2014;14:254.

14. Dewa CS, Loong D, Bonato S, et al. How does burnout affect physician productivity? A systematic literature review. BMC Health Serv Res 2014;14:325.

15. Shanafelt TD, Balch CM, Bechamps G, et al. Burnout and medical errors among American surgeons. Ann Surg 2010;251:995-1000.

16. Fahrenkopf AM, Sectish TC, Barger LK, et al. Rates of medication errors among depressed and burnt out residents: prospective cohort study. BMJ 2008;336:488-91.

17. Dyrbye LN, Varkey P, Boone SL, et al. Physician satisfaction and burnout at different career stages. Mayo Clin Proc 2013;88:1358-67.

18. Welp A, Meier LL, Manser T. Emotional exhaustion and workload predict clinician-rated and objective patient safety. Front Psychol 2014;5:1573.

19. Aiken LH, Clarke SP, Sloane DM, et al. Hospital nurse staffing and patient mortality, nurse burnout, and job dissatisfaction. JAMA 2002;288:1987-93.

20. Krasner MS, Epstein RM, Beckman H, et al. Association of an educational program in mindful communication with burnout, empathy, and attitudes among primary care physicians. JAMA 2009;302:1284-93.

21. Amutio A, Martínez-Taboada C, Hermosilla D, et al. Enhancing relaxation states and positive emotions in physicians through a mindfulness training program: a one-year study. Psychol Health Med 2015;20:720-31.

22. Asuero AM, Queraltó JM, Pujol-Ribera E, et al. Effectiveness of a mindfulness education program in primary health care professionals: a pragmatic controlled trial. J Contin Educ Health Prof 2014;34:4-12.

23. West CP, Dyrbye LN, Rabatin JT, et al. Intervention to promote physician well-being, job satisfaction, and professionalism: a randomized clinical trial. JAMA Intern Med 2014;174:527. 
24. West CP, Dyrbye LN, Satele D, et al. A randomized controlled trial evaluating the effect of COMPASS (COlleagues Meeting to Promote and Sustain Satisfaction) small group sessions on physician wellbeing, meaning, and job satisfaction. J Gen Intern Med 2015;30.

25. Panagioti M, Panagopoulou E, Bower P, et al. Controlled interventions to reduce burnout in physicians: a systematic review and meta-analysis. JAMA Intern Med 2017;177:195-205.

26. Seligman ME, Steen TA, Park N, et al. Positive psychology progress: empirical validation of interventions. Am Psychol 2005;60:410-21.

27. Gander F, Proyer RT, Ruch W, et al. Strength-based positive interventions: further evidence for their potential in enhancing well-being and alleviating depression. J Happiness Stud 2013;14:1241-59.

28. Sin NL, Lyubomirsky S. Enhancing well-being and alleviating depressive symptoms with positive psychology interventions: a practice-friendly meta-analysis. J Clin Psychol 2009;65:467-87.

29. Bianchi R, Laurent E. Emotional information processing in depression and burnout: an eye-tracking study. Eur Arch Psychiatry Clin Neurosci 2015;265:27-34.

30. Bianchi R, Schonfeld IS, Laurent E. Is it time to consider the "burnout syndrome" a distinct illness? Front Public Health 2015;3.

31. Wheeler DL, Vassar M, Worley JA, et al. A reliability generalization meta-analysis of coefficient alpha for the maslach burnout inventory. Educ Psychol Meas 2011;71:231-44.

32. Schaufeli WB, Bakker AB, Hoogduin $\mathrm{K}$, et al. on the clinical validity of the maslach burnout inventory and the burnout measure. Psychol Health 2001;16:565-82.

33. Sexton JB, Adair KC, Leonard MW, et al. Providing feedback following Leadership WalkRounds is associated with better patient safety culture, higher employee engagement and lower burnout. BMJ Qual Saf 2018;27:bmjqs-2016-006399:261-70

34. Profit J, Sharek PJ, Amspoker AB, et al. Burnout in the NICU setting and its relation to safety culture. BMJ Qual Saf 2014;23:806-13.

35. Andresen EM, Malmgren JA, Carter WB, et al. Screening for depression in well older adults: evaluation of a short form of the CES-D (Center for Epidemiologic Studies Depression Scale). Am J Prev Med 1994;10:77-84

36. Björgvinsson T, Kertz SJ, Bigda-Peyton JS, et al. Psychometric properties of the CES-D-10 in a psychiatric sample. Assessment 2013;20:429-36.

37. Lin JD, Lin PY, Wu CL. Wellbeing perception of institutional caregivers working for people with disabilities: use of subjective happiness scale and satisfaction with life scale analyses. Res Dev Disabil 2010;31:1083-90.

38. Lyubomirsky S, Lepper HS. A measure of subjective happiness: preliminary reliability and construct validation. Soc Indic Res 1999;46:137-55

39. Sexton JB, Schwartz SP, Chadwick WA, et al. The associations between work-life balance behaviours, teamwork climate and safety climate: cross-sectional survey introducing the work-life climate scale, psychometric properties, benchmarking data and future directions. BMJ Qual Saf 2016:bmjqs-2016-006032.
40. Wellenzohn S, Proyer RT, Ruch W. How do positive psychology interventions work? A short-term placebo-controlled humor-based study on the role of the time focus. Pers Individ Dif 2016;96:1-6.

41. Peterson RA. A Meta-analysis of cronbach's coefficient alpha. J Consum Res 1994;21:381-91.

42. Sawilowsky S. New effect size rules of thumb. Theor Behav Found Educ Fac Publ 2009

43. McAllister-Williams RH. Do antidepressants work? A commentary on "initial severity and antidepressant benefits: a meta-analysis of data submitted to the food and drug administration" by Kirsch et al. Evid Based Ment Health 2008;11:66-8.

44. Froh JJ, Kashdan TB, Ozimkowski KM, et al. Who benefits the most from a gratitude intervention in children and adolescents? Examining positive affect as a moderator. J Posit Psychol 2009;4:408-22.

45. Demerouti E, Bakker AB, Nachreiner F, et al. The job demandsresources model of burnout. J Appl Psychol 2001;86:499-512.

46. Tugade MM, Fredrickson BL. Resilient individuals use positive emotions to bounce back from negative emotional experiences. $J$ Pers Soc Psychol 2004;86:320-33.

47. Fredrickson BL, Mancuso RA, Branigan C, et al. The undoing effect of positive emotions. Motiv Emot 2000;24:237-58.

48. Tice DM, Baumeister RF, Shmueli D, et al. Restoring the self: Positive affect helps improve self-regulation following ego depletion. $J$ Exp Soc Psychol 2007;43:379-84.

49. Fredrickson BL. The role of positive emotions in positive psychology. The broaden-and-build theory of positive emotions. Am Psychol 2001;56:218-26.

50. Rippstein-Leuenberger K, Mauthner O, Sexton JB, et al. Three good themes: a qualitative analysis of the three good things intervention in health care workers. Rev 2017.

51. United States Department of Labor. Employed persons by detailed occupation, sex, race, and hispanic or latino ethnicity: United States Department of Labor.

52. Abbott J-A, Klein B, Hamilton C, et al. The impact of online resilience training for sales managers on wellbeing and performance. E-Journal of Applied Psychology 2009;5:89-95.

53. Mongrain M, Anselmo-Matthews T. Do positive psychology exercises work? A replication of Seligman et al. (2005). J Clin Psychol 2012;68:382-9.

54. Colombo O, Ferretti VV, Ferraris C, et al. Is drop-out from obesity treatment a predictable and preventable event? Nutr J 2014;13:13.

55. Dalle Grave R, Calugi S, Compare A, et al. Weight loss expectations and attrition in treatment-seeking obese women. Obes Facts 2015;8:311-8.

56. Bahadir A, lliaz S, Yurt S, et al. Factors affecting dropout in the smoking cessation outpatient clinic. Chron Respir Dis 2016:13:155-61.

57. Geraghty AW, Torres LD, Leykin Y, et al. Understanding attrition from international Internet health interventions: a step towards global eHealth. Health Promot Int 2013;28:442-52.

58. Eysenbach G. The law of attrition. J Med Internet Res 2005;7:e11. 